\title{
Mapping Linear Workflows with Computation/Communication Overlap
}

\author{
Kunal Agrawal ${ }^{1}$, Anne Benoit ${ }^{*, 2}$ and Yves Robert ${ }^{2}$ \\ ${ }^{1}$ CSAIL, Massachusetts Institute of Technology, USA \\ ${ }^{2}$ LIP, École Normale Supérieure de Lyon, France \\ kunal_ag@mit.edu, \{Anne.Benoit|Yves.Robert\}@ens-lyon.fr
}

\begin{abstract}
This paper presents theoretical results related to mapping and scheduling linear workflows onto heterogeneous platforms. We use a realistic architectural model with bounded communication capabilities and full computation/communication overlap. This model is representative of current multi-threaded systems. In these workflow applications, the goal is often to maximize throughput or to minimize latency. We present several complexity results related to both these criteria. To be precise, we prove that maximizing the throughput is NP-complete even for homogeneous platforms and minimizing the latency is NP-complete for heterogeneous platforms. Moreover, we present an approximation algorithm for throughput maximization for linear chain applications on homogeneous platforms, and an approximation algorithm for latency minimization for linear chain applications on all platforms where communication is homogeneous (the processor speeds can differ). In addition, we present algorithms for several important special cases for linear chain applications. Finally, we consider the implications of adding feedback loops to linear chain applications.
\end{abstract}

Key words: pipeline graphs, workflow, scheduling, mapping, period, latency, feedback loop, complexity results.

\section{INTRODUCTION}

Pipelined workflows are a popular programming paradigm for streaming applications like video and audio encoding and decoding, DSP applications etc. Streaming applications are becoming increasingly prevalent, and many languages are being continually designed to support these applications (see [1] for survey of older streaming languages). In these languages, the programmer expresses its programs by creating a workflow graph, and the system maps this workflow graph on a target machine.

A workflow graph contains several stages, and these stages are connected to each other using first-infirst-out channels. Data is input into the graph using input channel(s) and the outputs are produced on the

* Corresponding author: A. Benoit, LIP, ENS Lyon, 46 allée d'Italie, 69364 Lyon Cedex 07, France, Phone +33472728758 , Fax +33 472728080, Email Anne. Benoiteens-lyon.fr. 
output channel(s). Since data continually flows through these applications, the goal of a scheduler is often to increase the throughput and/or decrease the latency. One can think of these applications as a graph, where stages are the nodes and the channels are the edges. Most of the problems related to mapping general graphs optimally are rather obviously NP-Complete; therefore, we consider the special case of linear chains where they may or may not have feedback edges. In this domain, we provide comprehensive theoretical analysis of the complexity of mapping these graphs on both homogeneous and heterogeneous distributed memory platforms.

Subhlok and Vondran [2] studied the problem of mapping linear chain graphs on homogeneous platforms, and these complexity results were extended for heterogeneous platforms under the one-port model in [3]. In this model, the processor can either compute, receive an incoming communication, or send an outgoing communication at a time. This model does a good job of representing single-threaded systems. Unfortunately, this model is not suitable for handling general mappings, or applications which have feedback, and it can deadlock in this case. Therefore, in this paper, we explore the bounded-multiport model, which allows multiple incoming and outgoing communications simultaneously, and allows the computation and communication to overlap. To the best of our knowledge, the bounded-multiport model with overlap has not been explored for linear chains, and we start to explore its complexity before extending the approach to applications with feedback.

We consider three kinds of platforms for mapping these applications. Fully Homogeneous platforms are those which have identical processors. That is, all processors run at the same speed, and communicate with each other using links of the same bandwidth. Communication Homogeneous platforms are those in which the processors may have different speeds, but they are all connected by identical communication interconnect. Fully Heterogeneous platforms are those where both processor speeds and the speed of the interconnect changes from processor to processor.

Here is the summary of our results:

- Finding the mapping with optimal throughput is NP-complete for all platforms in the bounded multiport model with overlap. Finding the mapping with optimal latency is NP-complete for Communication Homogeneous platforms. The problem of finding the mapping with optimal latency for Fully Homogeneous platforms is left open. These proofs are given in Section III.

- Section IV shows finding the best interval mappings for both throughput and latency can be done 
in polynomial time for Fully Homogeneous platforms. These interval mappings are commonly used since they minimize the communication overhead. However, finding optimal interval mappings is NP-complete for Communication Homogeneous platforms.

- Since finding the best mapping is NP-complete, we looked for approximation algorithms. In Section V, we show that interval mappings provide a 2-approximation for throughput on Fully Homogeneous platforms. Since these interval mappings can be found in polynomial time, we have a 2-approximation algorithm for the throughput. Mapping all the stages on the same processor provides a 1.5-approximation for latency on Communication Homogeneous platforms (hence also for Fully Homogeneous platforms). However, this result does not hold for Fully Heterogeneous platforms.

- In the special case where an infinite number of processors are available to the application, interval mappings are optimal for throughput. Similarly, interval mappings are optimal for throughput if the application is regular — all the stages perform the same amount of computation and communication. Therefore, for both these special cases, we have polynomial time algorithms for linear chains on Fully Homogeneous platforms for throughput. These results are explained in Section VI.

- In Section VII, we show that almost all problems become more difficult when feedback loops are added. Interval mappings are no longer a good approximation for throughput even for Fully Homogeneous platforms. However, mapping all stages on the same processor is still a good approximation for latency for Communication Homogeneous platforms. In addition, we prove that optimizing throughput is NP-complete even for infinite number of processors when we have feedback loops.

Due to lack of space, all the proofs have not been included. In addition, some of the related work has not been mentioned in this section. Please refer to the extended version [4] for full proofs and more related work.

\section{FRAMEWORK}

In this section we first describe the application model and architectural framework. Then we detail mapping rules and objectives.

\section{A. Application Model}

We consider simple application workflows whose graphs are a pipeline (i.e., a linear chain). Such graphs are representative of a wide class of applications, and constitute the typical building blocks upon 


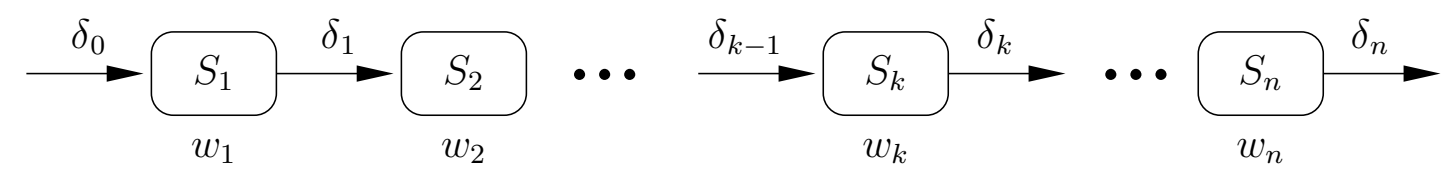

Fig. 1. Application linear chain.

which to build and execute more complex workflows. A pipeline graph of $n$ stages $S_{k}, 1 \leq k \leq n$ is illustrated on Figure 1. Consecutive data sets are fed into the pipeline and processed from stage to stage, until they exit the pipeline after the last stage.

Each stage executes a task. More precisely, the $k$-th stage $S_{k}$ receives an input from the previous stage, of size $\delta_{k-1}$, performs a number of $w_{k}$ computations, and outputs data of size $\delta_{k}$ to the next stage. This operation corresponds to the $k$-th task and is repeated periodically on each data set. Communications and computations are done in parallel, thus input for data set $i+1$ is received while computing for data set $i$ and sending result for data set $i-1$. The first stage $S_{1}$ receives an input of size $\delta_{0}$ from the outside world, while the last stage $S_{n}$ returns the result, of size $\delta_{n}$, to the outside world.

\section{B. Execution Model}

1) Platform Graph: We target a heterogeneous platform with $p$ processors $P_{u}, 1 \leq u \leq p$, fully interconnected as a (virtual) clique. There is a bidirectional link link $k_{u, v}: P_{u} \rightarrow P_{v}$ between any processor pair $P_{u}$ and $P_{v}$, of bandwidth $b_{u, v}$. Note that we do not need to have a physical link between any processor pair. Instead, we may have a switch, or even a path composed of several physical links, to interconnect $P_{u}$ and $P_{v}$; in the latter case we would retain the bandwidth of the slowest link in the path for the value of $b_{u, v}$. In the most general case, we have fully heterogeneous platforms, with different processors speeds and link capacities. The speed of processor $P_{u}$ is denoted as $s_{u}$, and it takes $X / s_{u}$ time-units for $P_{u}$ to execute $X$ floating point operations. We also enforce a linear cost model for communications, hence it takes $X / b_{u, v}$ time-units to send (resp. receive) a message of size $X$ to (resp. from) $P_{v}$.

Finally, there are additional constraints on the communications: in addition to link bandwidths, we deal with processor network cards and we bound the total communication capacity of each computing resource. We denote by $\mathrm{B}_{u}^{i}$ (resp. $\mathrm{B}_{u}^{o}$ ) the capacity of the input (resp. output) network card of processor $P_{u}$. In other words, $P_{u}$ cannot receive more than $1 / \mathrm{B}_{u}^{i}$ data items per time-unit, and it cannot send more than $1 / \mathrm{B}_{u}^{o}$ data items per time-unit. 
We classify particular cases which are important, both from a theoretical and practical perspective. Fully Homogeneous platforms have identical processors $\left(s_{u}=s\right)$ and homogeneous communication devices $\left(b_{u, v}=b\right.$ for link bandwidths, and $\mathrm{B}_{u}^{i}=\mathrm{B}^{i}, \mathrm{~B}_{u}^{o}=\mathrm{B}^{o}$ for network cards). They represent typical parallel machines. Communication Homogeneous platforms are still interconnected with homogeneous communication devices, but they have different-speed processors $\left(s_{u} \neq s_{v}\right)$. They correspond to networks of workstations with plain TCP/IP interconnects or other LANs. Finally, Fully Heterogeneous platforms are the most general, fully heterogeneous architectures. Hierarchical platforms made up with several clusters interconnected by slower backbone links can be modeled this way.

Finally, we assume that two special additional processors $P_{\text {in }}$ and $P_{\text {out }}$ are devoted to input/output data. Initially, the input data for each task resides on $P_{i n}$, while all results must be returned to and stored in $P_{\text {out }}$. Of course we may have a single processor acting as the interface for the computations, i.e., $P_{\text {in }}=P_{\text {out }}$.

2) Realistic Communication Models: The standard model for DAG scheduling heuristics [5] does a poor job to model physical limits of interconnection networks. The model assumes an unlimited number of simultaneous sends and receives, i.e., a network card of infinite capacity, on each processor. A more realistic model is the one-port model [6]. In this model, a given processor can be involved in a single communication at any time-step, either a send or a receive. However, independent communications between distinct processor pairs can take place simultaneously. The one-port model seems to fit the performance of some current MPI implementations, which serialize asynchronous MPI sends as soon as message sizes exceed a few megabytes [7].

The one-port model fully accounts for the heterogeneity of the platform, as each link has a different bandwidth. It generalizes simpler models [8] where communication time only depends on the sender, not on the receiver. In these models, the communication speed from a processor to all its neighbors is the same. A study of mapping strategies for linear chain application graphs under the one-port model has been conducted in [3].

Another realistic model is the bounded multiport model [9]. In this model, the total communication volume outgoing from a given node is bounded (by the capacity of its network card), but several communications along different links can take place simultaneously (provided that the link bandwidths are not exceeded either). We point out that recent multi-threaded communication libraries such as 
MPICH2 [10] now allow for initiating multiple concurrent send and receive operations, thereby providing practical realizations of the multiport model.

3) Computation/Communication Overlap: Another key assumption to define the execution model is to decide whether computation can overlap with (independent) communication. Most state-of-the-art processors running a threaded operating system are indeed capable of such an overlap (even though it may be at the price of some degradation in the computing speed [11]).

The main emphasis of this paper is to investigate the complexity of various mapping problems under the bounded multiport model with computation/communication overlap. These two assumptions (multiport and overlap) fit well together because they both require a multi-threaded system. However, they turn out to have a tremendous impact on the definition of the throughput and of the latency that can be achieved: we need to drastically change the definitions that are used under the one-port model without overlap [2], [3]: see the example in Section II-C.1 below.

\section{Mapping Strategies}

Key metrics for a given workflow are the throughput and the latency. The throughput measures the aggregate rate of processing of data, and it is the rate at which data sets can enter the system. Equivalently, the inverse of the throughput, defined as the period, is the time interval required between the beginning of the execution of two consecutive data sets. The latency is the time elapsed between the beginning and the end of the execution of a given data set, hence it measures the response time of the system to process the data set entirely. Note that minimizing the latency is antagonistic to minimizing the period.

The mapping problem consists of assigning application stages to platform processors. Formally, we search for an allocation function of stages to processors, defined as $a:[1 . . n] \rightarrow[1 . . p]$. We always assume in the following that $a(0)=i n$ and $a(n+1)=o u t$.

There are several mapping strategies. The more restrictive mappings are one-to-one; in this case, each stage is assigned a different processor. Then the allocation function $a$ is a one-to-one function, and there must be at least as many processors as application stages. Another strategy is very common for linear chains: we may decide to group consecutive stages onto a same processor, in order to avoid some costly communications. However, a processor is only processing an interval of consecutive stages. Such a mapping is called an interval-based mapping. Finally, we can consider general mappings, for which 


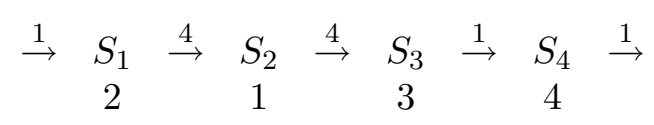

Fig. 2. Toy example to explain how period and latency are determined.

there is no constraint on the allocation function: each processor is assigned one or several stage intervals.

1) Working out an Example: Consider the little example of Figure 2 with four stages. Below each stage $S_{i}$ we have indicated the number of computations $w_{i}$ (expressed in flops) that it requires: $w_{1}=2$, $w_{2}=1, w_{3}=3$ and $w_{4}=4$. The value of each $\delta_{i}$ is indicated at the right of each stage: $\delta_{0}=1$, $\delta_{1}=\delta_{2}=4, \delta_{3}=\delta_{4}=1$. As for the platform, assume that we have a Fully Homogeneous platform with two identical processors $P_{1}$ and $P_{2}$ of speed $s=1$ and of network card capacities $\mathrm{B}^{i}=\mathrm{B}^{o}=1$, and with identical links of bandwidth $b=1$.

We can achieve a perfect load-balance of the computations if we map stages $S_{1}$ and $S_{3}$ on $P_{1}$, and stages $S_{2}$ and $S_{4}$ on $P_{2}$. What would be the period and the latency with such a mapping? Under the bounded multiport model with computation/communication overlap, we achieve a period $\mathcal{P}=5$. Indeed, $P_{1}$ has two incoming communications of size $\frac{\delta_{0}}{b}+\frac{\delta_{2}}{b}=5$, and we have $\frac{\delta_{0}+\delta_{2}}{\mathrm{~B}^{i}}=5$, so that all constraints (link bandwidths and network card capacity) are verified for incoming communications. Similarly, we check that for outgoing communications $\frac{\delta_{1}}{b}+\frac{\delta_{3}}{b}=\frac{\delta_{1}+\delta_{3}}{\mathrm{~B}^{\circ}}=5$. Finally, we chose the mapping so that $\frac{w_{1}+w_{3}}{s}=5$. Altogether, the cycle-time of processor $P_{1}$ is 5 , which means that it can start to process a new data set every 5 time units. We perform the same analysis for $P_{2}$ and derive that its cycle-time also is 5 . The period is the maximum of the cycle-times of the processors, hence we derive that $\mathcal{P}=5$.

Computing the latency is more complicated. At first sight, we might say that the latency is the longest path in the execution, of length $\frac{\delta_{0}}{b}+\frac{w_{1}}{s}+\frac{\delta_{1}}{b}+\frac{w_{2}}{s}+\frac{\delta_{2}}{b}+\frac{w_{3}}{s}+\frac{\delta_{3}}{b}+\frac{w_{4}}{s}+\frac{\delta_{4}}{b}=21$. However, this is only possible for the first data sets. See Figure 3: if the first data set $\mathbf{d s}^{(0)}$ enters the platform at time $t=0$, then $P_{1}$ is active at time $t=1$ and $t=2$ (for stage $S_{1}$ ), and then $t=12, t=13$, and $t=14$ (for stage $\left.S_{3}\right)$. If a new data set enters every five time-units to achieve a period $\mathcal{P}=5$, then data set $\mathrm{ds}^{(k)}$ enters at time $t=5 k$ and $P_{1}$ is active for it at time $t=1+5 k, 2+5 k, 12+5 k, 13+5 k, 14+5 k$. Because this holds true for all $k \geq 0$, we have many conflicts! For instance the first conflict is at $t=12$ for stage $S_{1}$ of $\mathrm{ds}^{(3)}$ and stage $S_{3}$ of $\mathrm{ds}^{(1)}$. Similarly, we obtain conflicts for $P_{2}$ and for the communication link from $P_{1}$ to $P_{2}$. 


$$
\begin{aligned}
& \text { in } \rightarrow P_{1} \\
& P_{1} \\
& P_{1} \rightarrow P_{2} \\
& P_{2} \rightarrow P_{1} \\
& P_{2} \\
& P_{2} \rightarrow \text { out }
\end{aligned}
$$

Fig. 3. Processing the first data set to achieve a latency $\mathcal{L}=21$ would lead to conflicts for the next data sets.

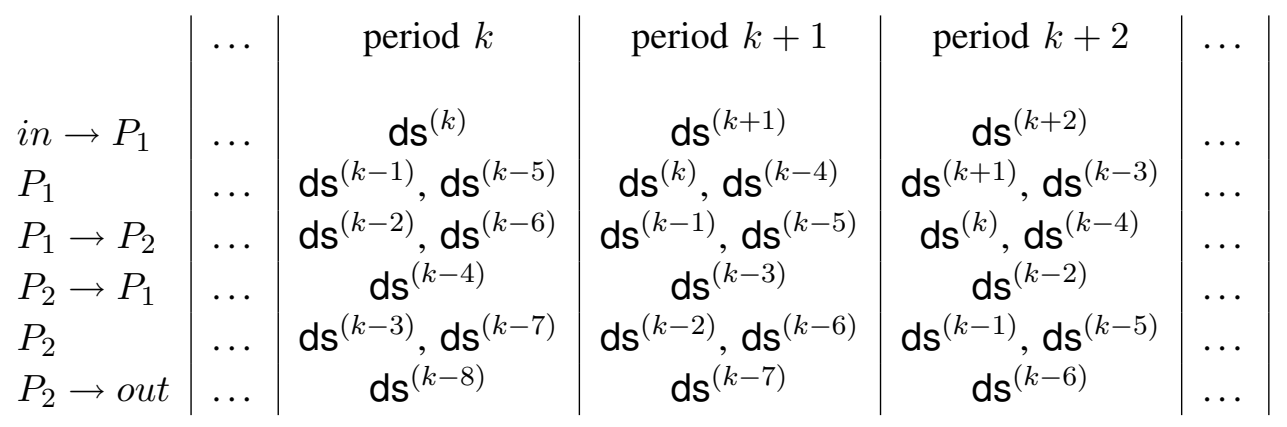

Fig. 4. Achieving a latency $\mathcal{L}=9 \mathcal{P}=45$ in steady state mode.

In fact, in steady-state we can only achieve a much larger latency: see Figure 4, where the latency is shown to be $\mathcal{L}=45$. The idea is simple: when a processor executes some computation for a data set $d s^{(k)}$, then it simultaneously receives input corresponding to data set $d s^{(k+1)}$ and performs output corresponding to data set $\mathrm{ds}^{(k-1)}$. In turn, the next processor operates on data set $\mathrm{ds}^{(k-2)}$, and so on. This example shows that a key parameter is the number of stage intervals in the mapping. Here we define an interval as a subset of consecutive stages that is not mapped onto the same processor as the previous stages (see Section II-C.3 for a more formal definition). In our example we have four intervals composed of 1 stage each, because each stage is mapped onto a different processor than its predecessor, hence $K=4$. If there are $K$ intervals, there are $K+1$ communication links, hence it takes $K+(K+1)=2 K+1$ periods for a data set to be processed entirely. We check in Figure 4 that we need $2 K+1=9$ periods to compute a data set, so that $\mathcal{L}=9 \times 5=45$.

Note that computing the latency with a non-overlap model looks very difficult. This is because each processor would have to decide which of its two incoming communications to execute first, and which of its two outgoing communications to execute first. Any choice is likely to increase the latency for some data set. The problem is similar for the one-port or the multiport model. Indeed, with the oneport model, we have no choice and must serialize the two communications. On the contrary, with the 
multiport model we can decide to execute both communications in parallel, but this is not helpful as it only delays the first communication without speeding up the second one. For both models it is hard to decide which communication to give priority to. A simple (greedy) algorithm would give priority to the communication involving the least recent data set. We could easily work out such an algorithm for our little example. However, computing a closed form expression for the latency in the general case seems untractable. Furthermore, we point out that without overlap the difficulty comes from the fact that several stage intervals are mapped onto the same processor, which requires to arbitrate between as many incoming (and outgoing) communications. If we enforce interval-based mappings (see Section II-C), then each processor is assigned a single interval and the computation for the latency is greatly simplified.

Note also that interval-based mappings are interesting for the multiport model as they allow to decrease the value of $K$, the number of stage intervals, which never exceeds the number $p$ of available processors for such mappings. However, general mappings may still be needed to better balance the work, hence to decrease the period, at the price of a larger value of $K$. Such trade-offs are at the heart of the algorithms and complexity proofs that follow. Finally, we point out that introducing feedback loops in Section VII will further complicate these issues.

2) Period: As illustrated in Figure 4, we assume that a new data set arrives every period at a regular pace. Let $P_{1}$ be the processor in charge of the first stage. While it is computing for data set $k$, it simultaneously receives input corresponding to data set $k+1$ and sends output corresponding to data set $k-1$. More precisely, the latter output is related to the first stage $S_{i}$ such that $a(i) \neq a(i+1)$ : for a given data set, all computations corresponding to stages $S_{1}$ to $S_{i}$ are performed during the same period. As in the example of Figure 2, $P_{1}$ can be assigned other stage intervals, and the period must be large enough so that the sum of all its computations does not exceed the value of the period. The same holds true for the sum of its incoming communications, and for the sum of its outgoing communications.

Formally, under the bounded multiport model with overlap, the cycle-time of processor $P_{u}, 1 \leq u \leq p$, 
is defined as:

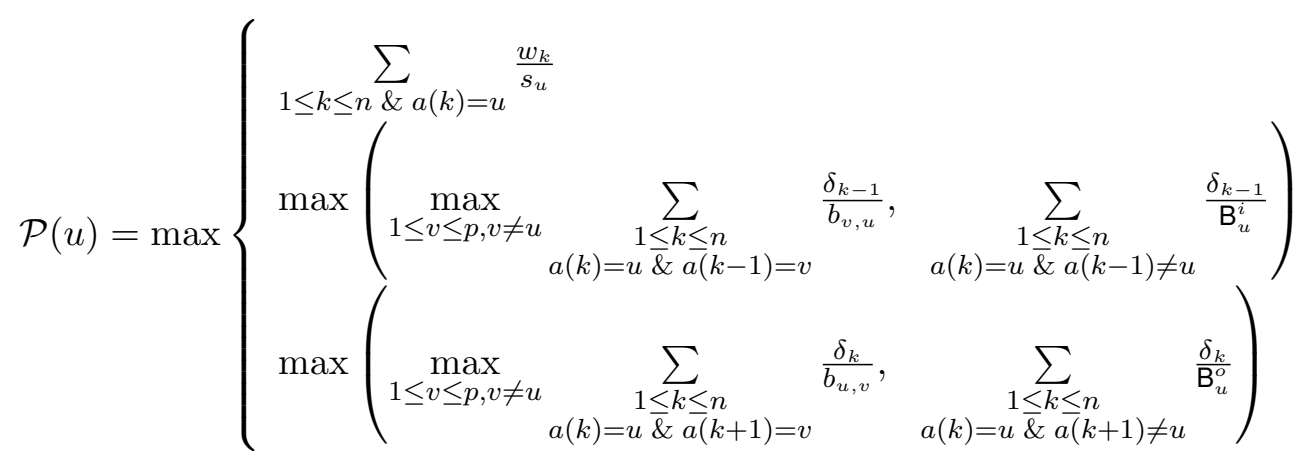

Line (1) is bounding the period when the computation step is the longest activity. It expresses the computation time for processor $P_{u}$. Line (2) represents the time for input communications, which is bounded by the links from incoming processors, and by the network card limit $\mathrm{B}_{u}^{i}$. We need to consider all stages $S_{k}$ that are assigned to $P_{u}$ but whose predecessor $S_{k-1}$ is not. We check that no link bandwidth is exceeded from any other processor $P_{v}$ and we account for all these communications together for the network card capacity of $P_{u}$. Similarly, line (3) deals with output communications. Also, notice that for the sake of simplicity, $i n=0$ and out $=n+1$. The period of the mapping is then $\mathcal{P}=\max _{1 \leq u \leq p} \mathcal{P}(u)$.

3) Latency: As stated in Section II-C.1, the latency depends on the number of stage intervals, or equivalently, of the number of changes from one processor to a different one. We define $K(i, j)$ as the number of stage intervals between stages $S_{i}$ and $S_{j}$, where $i<j$. Formally, $K(i, j)=\sum_{i \leq k<j \& a(k) \neq a(k+1)} 1$. The total number of intervals in the pipeline is $K=K(1, n+1)$. Since we always have $a(n+1)=$ out $\neq a(n)$, $K \geq 1\left(K=1\right.$ if all stages are mapped onto the same processor $\left.P_{a(n)}\right)$. Again, we point out that $K$ depends on the number of processor changes, and thus it is increased if a processor is in charge of several distinct stage intervals. The latency is then defined as $2 K+1$ times the period, since a data set traverses the whole pipeline in $2 K+1$ time-steps, and each time-step has a duration of $\mathcal{P}: \mathcal{L}=(2 K+1) \times \mathcal{P}$.

Consider again the toy example of Figure 2. With the chosen mapping, $\mathcal{P}=5$ but $K=9$, hence $\mathcal{L}=45$. The value of the period is optimal because the sum of the four computation weights is 10 , and we have two processors of speed 1. But the value of the latency is not optimal. For instance, assign all stages to the same processor: the period becomes $\mathcal{P}=10$ but now $K=3$, hence $\mathcal{L}=30$. We can also assign the first three stages to $P_{1}$ and the last one to $P_{2}$ : then we derive $\mathcal{P}=6, K=5$ and $\mathcal{L}=30$ too. 


\section{Complexity Results}

This section provides complexity results for period and latency minimization.

Theorem 1: On Fully Homogeneous platforms, finding the general mapping which minimizes the period is NP-complete.

Proof: The proof uses a reduction from 2-PARTITION. See [4] for details.

Like the period minimization problem, we believe that latency minimization is also NP-complete. In Section IV, we shall see that optimal interval mappings can be found in polynomial time. However, unfortunately, interval mappings are not guaranteed to be optimal for latency either. The following example shows that interval mappings are not optimal for latency for Fully Homogeneous platforms. Consider 150 homogeneous processors with speeds all equal to 1 . The application pipeline contains 300 stages as shown below, and there are no communications.

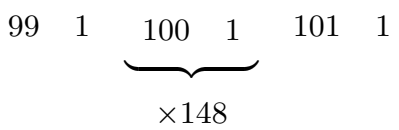

In other words, the first stage has work 99 , the second stage has work 1 . The third and the fourth stages have work 100 and 1 respectively. These third and fourth stages are repeated 148 times (the fifth stage has work 100, sixth stage 1, and so on). The 299-th stage has work 101 and the last stage has work 1 again. The best schedule is perfectly load balanced but not interval-based: put stages 1,2 , and 300 on one processor, all pairs of 100 and 1 on distinct 148 processors, and then stage 299 with work 101 on the last processor. The period of this mapping is 101 and the latency is 30603. The best interval-based schedule uses 75 intervals with period 203, and latency 30653 (we used the dynamic programming algorithm of Section IV to obtain this result).

Since minimum latency mapping may not be an interval-based mapping, it appears as though latency minimization is in fact NP-complete for Fully Homogeneous platforms. However, the proof has not been forthcoming, and this problem is left open. The next theorem shows that minimizing latency is NP-complete for Communication Homogeneous platforms.

Theorem 2: On Communication Homogeneous platforms, finding the general mapping which minimizes the latency is NP-complete. 
Proof: Recall that the latency for a general mapping is defined as $\mathcal{L}=(2 q+1) \mathcal{P}_{q}$, where $q$ is the number of intervals in the mapping, and $\mathcal{P}_{q}$ the period, i.e., the maximum cycle-time of any processor involved in the execution. If a given processor is assigned several (non-consecutive) intervals, its cycletime is the maximum of the three following costs: (i) sum of its incoming communications (one per interval); (ii) sum of its outgoing communications (one per interval); and (iii) sum of its computations (all its assigned stages).

Let LATENCY-DEC denote the decision problem: given an application pipeline with $n$ stages, a Communication Homogeneous platform with $p$ processors, and a bound $\mathrm{L}$, does there exist a mapping whose latency is not greater than L? The LATENCY-DEC problem clearly belongs to the class NP: given a mapping of the stages onto $p$, it is easy to compute which intervals are mapped on the same processor, and to compute the maximum cycle-time $\mathcal{P}_{q}$ of all the $p$ processors and to check that $(2 q+1) \mathcal{P}_{q} \leq \mathrm{L}$.

To establish the completeness, we use a reduction from NUMERICAL MATCHING WITH TARGET SUMS (NMWTS), which is NP-complete in the strong sense [12]. We consider an instance $\mathfrak{I}_{1}$ of NMWTS: given $3 m$ numbers $x_{1}, x_{2}, \ldots, x_{m}, y_{1}, y_{2}, \ldots, y_{m}$ and $z_{1}, z_{2}, \ldots, z_{m}$, does there exist two permutations $\sigma_{1}$ and $\sigma_{2}$ of $\{1,2, \ldots, m\}$, such that $x_{i}+y_{\sigma_{1}(i)}=z_{\sigma_{2}(i)}$ for $1 \leq i \leq m$ ? Because NMWTS is NP-complete in the strong sense, we can encode the $3 m$ numbers in unary and assume that the size of $\mathfrak{I}_{1}$ is $O(m+M)$, where $M=\max _{i}\left\{x_{i}, y_{i}, z_{i}\right\}$. We also assume that $\sum_{i=1}^{m} x_{i}+\sum_{i=1}^{m} y_{i}=\sum_{i=1}^{m} z_{i}$, otherwise $\mathfrak{I}_{1}$ cannot have a solution.

We build the following instance $\mathfrak{I}_{2}$ of LATENCY-DEC. We define $n=(M+3) m$ stages, whose computation weights $w_{i}$ are outlined below:

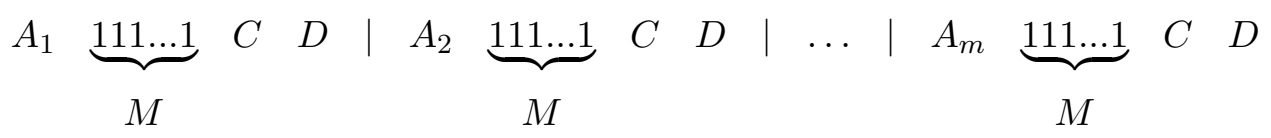

Here, we introduce $U=72 \mathrm{~m}^{2}$ (see below where this value comes from) and we let $B=(U+2) M$, $C=B+3 M, D=B+5 M$, and $A_{i}=B+x_{i}$ for $1 \leq i \leq m$. To define the $w_{i}$ formally for $1 \leq i \leq n$, let $N=M+3$. We have for $1 \leq i \leq m: w_{(i-1) N+1}=A_{i}=B+x_{i}, w_{(i-1) N+j}=1$ for $2 \leq j \leq M+1$, $w_{i N-1}=C$, and $w_{i N}=D$. For the communication weights, we let $\delta_{i}=1$ for all the $n$ stages We define a platform with $p=3 m$ processors and homogeneous links of bandwidth $b=1$, As for the speeds, we let $s_{i}$ be the speed of processor $P_{i}$ where, for $1 \leq i \leq m: s_{i}=B+z_{i}, s_{m+i}=C+M-y_{i}$, and 
$s_{2 m+i}=D$. Finally, we ask whether there exists a solution matching the bound $\mathrm{L}=6 m+1$. Clearly, the size of $\mathfrak{I}_{2}$ is polynomial in the size of $\mathfrak{I}_{1}$. We now show that instance $\mathfrak{I}_{1}$ has a solution if and only if instance $\mathfrak{I}_{2}$ does.

Suppose first that $\mathfrak{I}_{1}$ has a solution, with permutations $\sigma_{1}$ and $\sigma_{2}$ such that $x_{i}+y_{\sigma_{1}(i)}=z_{\sigma_{2}(i)}$. For $1 \leq i \leq m$ :

- We map each stage $A_{i}$ and the following $y_{\sigma_{1}(i)}$ stages of weight 1 onto processor $P_{\sigma_{2}(i)}$.

- We map the following $M-y_{\sigma_{1}(i)}$ stages of weight 1 and the next stage, of weight $C$, onto processor $P_{m+\sigma_{1}(i)}$.

- We map the next stage, of weight $D$, onto processor $P_{2 m+i}$.

We have a partition of all the stages into $p=3 m$ intervals. For $1 \leq i \leq m$, the load and speed of the processors are indeed equal:

- The load of $P_{\sigma_{2}(i)}$ is $A_{i}+y_{\sigma_{1}(i)}=B+x_{i}+y_{\sigma_{1}(i)}$ and its speed is $B+z_{\sigma_{2}(i)}$.

- The load of $P_{m+\sigma_{1}(i)}$ is $M-y_{\sigma_{1}(i)}+C$, which is equal to its speed.

- The load and speed of $P_{2 m+i}$ are both $D$.

Each processor is assigned a single interval, hence the cost of its incoming and outgoing communications is equal to 1 . The mapping achieves a period $P_{p}=1$, and a latency $\mathcal{L}=(2 p+1) P_{p}=6 m+1=\mathrm{L}$, hence a solution to $\mathfrak{I}_{2}$.

Suppose now that $\mathfrak{I}_{2}$ has a solution, i.e., a mapping matching the latency bound $L=6 m+1$. Then we can show that this mapping is interval-based, and that it implies that $\mathfrak{I}_{1}$ has a solution. But this part of the proof is involved and technical: due to lack of space, please refer to [4].

Corollary 1: On Communication Homogeneous platforms, finding an interval mapping which minimizes the latency is NP-complete.

Proof: See the proof for Theorem 2.

\section{Algorithms for Interval-BASEd Mappings}

This section gives a polynomial time algorithm returning the best interval-based mapping, both for period and latency minimization problems on a Fully Homogeneous platform.

Theorem 3: On a Fully Homogeneous platform, finding the interval-based mapping which minimizes the period or the latency can be constructed in polynomial time. 
Proof: We provide a dynamic programming to build an interval-based mapping which minimizes the period or the latency. Let $P(i, u)$ be the best period that can be achieved by mapping stages $S_{1}$ to $S_{i}$ onto at most $u$ processors. The recurrence writes as follows:

$$
P(i, u)=\min _{0 \leq j<i}\left\{\max \left\{P(j, u-1), \frac{\sum_{k=j+1}^{i} w_{k}}{s}, \frac{\delta_{i}}{b}\right\}\right\}
$$

with the initializations, for $u \geq 0$ and $i>0$ :

$$
P(0, u)=\frac{\delta_{0}}{b} \quad \text { and } \quad P(i, 0)=+\infty
$$

In fact, we either map all the stages onto the same processor $(j=0$ in the min), or we cut the stages at an arbitrary place $(0<j<i)$ and allocate stages $j+1$ to $i$ to a single processor. The period is then the maximum between computations $\left(\sum w_{k}\right)$ and communications (output $\delta_{i} / b$ ). The input communication is taken into account in the term $P(j, u-1)$, since the output from stage $j$ is identical to the input to stage $j+1$. The first input communication is counted as $P(0, u)$. If there are not enough processors and we still have stages to process, we reach the case $P(i, 0)$ with $i>0$ which is not feasible, thus leading to a period of $+\infty$. For the period minimization problem, we compute $P(n, p)$, as any solution uses at most $p$ processors. For the latency minimization problem, we need to compute $\min _{1 \leq K \leq p}(2 K+1) \cdot P(n, K)$. The latency depends on the number of cuts, so it may be better to use less processors to decrease the $2 K+1$ value. Notice that if the solution returned by $P(n, K)$ uses strictly less than $K$ processors, the result will be reproduced with a smaller $K$, leading to a better latency, and thus the first solution will not be taken into account.

\section{ApProximation Algorithms for Period ANd LATENCY}

Theorem 4: Some interval-based mapping provides a 2-approximation for the optimal period for Fully Homogeneous platforms.

Proof: In this proof, given an optimal mapping, we generate an interval-based mapping that has at most twice the period.

Consider the best general mapping with $k$ intervals. If the best mapping has fewer than $p$ intervals, then we are done, since we cannot increase the period by giving each processor a distinct interval, and 
we have enough processors to do so.

Hence let us consider the case where $k>p$. Let the period of this optimal mapping be $P_{o}$. For every interval $i$, where $1 \leq i \leq k$, let $\Delta_{i}$ be the incoming communication, and $\Sigma_{i}$ be the computation requirements of interval $i\left(\Delta_{i+1}\right.$ is the outgoing communication for stage $i$ ). Without loss of generality, assume that for all processors $u$, we have $s_{u}=\mathrm{B}_{u}^{i}=\mathrm{B}_{u}^{o}=1$, and for all processor pairs $u, v, b_{u, v}=1$. Therefore, for all $i$, we have $\Delta_{i} \leq P_{o}$, and $\Sigma_{i} \leq P_{o}$. In addition, we know that $\sum_{i=1}^{k} \Sigma_{i} \leq p P_{o}$, since that is the maximum amount of computation $p$ processors can perform in $P_{o}$ time.

Now, we generate an interval-based mapping. We merge the consecutive intervals of the general mapping into larger intervals, called partitions. If a partition $r$ contains intervals $j$ to interval $l$, then $\sum_{i=j}^{l-1} \Sigma_{i}<P_{o}$ and $\sum_{i=j}^{l} \Sigma_{i} \geq P_{o}$. The first partition starts at interval 1 , and the last one ends at interval $k$. There are at most $p$ partitions, since the total computation is $\sum_{i=1}^{k} \Sigma_{i} \leq p P_{o}$ and each partition does at least $P_{o}$ computation.

Since we know that all intervals $i$ have $\Sigma_{i} \leq P_{o}$, we know that for any partition $r$ that contains intervals $j$ to $l$, we have $\sum_{i=j}^{l} \Sigma_{i} \leq 2 P_{o}$. In addition, the incoming communication for partition $r$ is $\Delta_{r}=\Delta_{j} \leq P_{o}$ and the outgoing communication for partition $r$ is $\Delta_{r+1}=\Delta_{l+1} \leq P_{o}$. Therefore, for every partition $r$, we have $\Sigma_{r} \leq 2 P_{o}, \Delta_{r} \leq P_{o}$ and $\Delta_{r+1} \leq P_{o}$. Therefore, each of these partitions can be mapped on different processors, to get an interval-based mapping with period at most $2 P_{o}$.

Unfortunately, interval mappings are not a constant-approximation for the period on Communication Homogeneous platforms. Here is an example to demonstrate this fact. Consider a Communication Homogeneous platform with $n+1$ processors; processor 1 has speed $s_{1}=2 K$ and the other $n$ processors have speed 1 , and $n>K^{2}$. Now consider an application as follows:

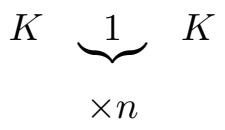

In other words, the first and the last stages have a computation of $K$, and there are $n$ intermediate stages which have a computation of 1 . In this example, a non-interval schedule would map the first and the last stages to processor 1 , and the remaining stages (one each) to the $n$ processors with speed 1 , generating a period of 1 . Unfortunately, any interval schedule has a period of at least $K$ since $n>K^{2}$. Therefore, the interval schedule can be as bad as a $K$-approximation at best for the period. 
Theorem 5: A mapping which puts all the stages on the fastest processor provides a 1.5-approximation for optimal latency on Communication Homogeneous platforms.

Proof: Consider the optimal mapping, which uses $k$ processors. Say that the latency with this mapping is $L_{o}$, and the period with this mapping is $P_{o}$. Since there are at least $k$ intervals in this mapping, we have $L_{o} \geq(2 k+1) P_{o}$. Now let the latency and period when all the stages are mapped on one processor be $L_{1}$ and $P_{1}$ respectively. We know that $P_{1} \leq k P_{o}$. Since $L_{1}=3 P_{1}$, we have $L_{1}=3 P_{1} \leq 3 k P_{o} \leq 3 k L_{o} /(2 k+1) \leq 1.5 L_{o}$.

Unfortunately, mapping all stages on the fastest processor does not provide a constant approximation for optimum latency for Fully Heterogeneous platforms, see [4] for counterexample.

\section{SPECiAl CASES}

Since both period and latency minimization appear to be NP-complete even on Fully Homogeneous platforms, we consider some special cases where one can in fact find polynomial time algorithms.

\section{A. Infinite Number of Processors}

Lemma 1: With an infinite number of processors on a Fully Homogeneous platform, there is an optimal interval-based mapping for both problems, (i) minimizing the period, and (ii) minimizing the latency.

Proof: This can be proved easily with an exchange argument. If processor $P_{u}$ is not handling a single interval of stages, since we have an infinite number of processors, we can give each interval of stages processed by $P_{u}$ to a new processor. Both the period and the latency can only decrease, since the new processors have less computation to perform than $P_{u}$, communications have not changed, and the number of distinct intervals in the mapping $K$ is still the same.

Theorem 6: With an infinite number of processors on a Fully Homogeneous platform, finding the general mapping which minimizes the period or the latency can be done in polynomial time.

Proof: This theorem is a direct consequence from Lemma 1 and Theorem 3. Indeed, Theorem 3 provides the best interval-based mapping, and Lemma 1 ensures that this interval-based mapping is better than any general mapping.

Also we notice that with a fixed number of processors, still on Fully Homogeneous platforms, Lemma 1 is not true anymore. Indeed, we can build an example in which the best latency is obtained with a general mapping which is not interval-based. Please refer to Section III for such an example. 


\section{B. Uniform Applications}

Now, let us consider a regular application, i.e., $\forall 1 \leq i \leq n, w_{i}=w$ and $\delta_{i}=\delta$, on a Communication Homogeneous platform. In this particular case, we have a lemma similar to Lemma 1: there is always an optimal mapping for latency which is interval-based. This can be easily proved using an exchange argument. Then, we can write a dynamic programming algorithm which returns the optimal period and latency in polynomial time. This algorithm is close to the one displayed in the proof of Theorem 3 (Section IV), and the interested reader may refer to [4] for details.

\section{Additional Complexity of FEedBack LoOpS}

Most of previous problems are already NP-hard, except some particular cases. Some of these special polynomial cases become NP-difficult when adding the extra complexity of feedback loops, as for instance the period minimization problem with an infinite number of processors. Also, some of the approximation results do not hold anymore.

Before revisiting previous complexity results, we formalize the feedback loops model and explain how period and latency should be computed so as to take feedback loops into account.

\section{A. Model for Feedback Loops}

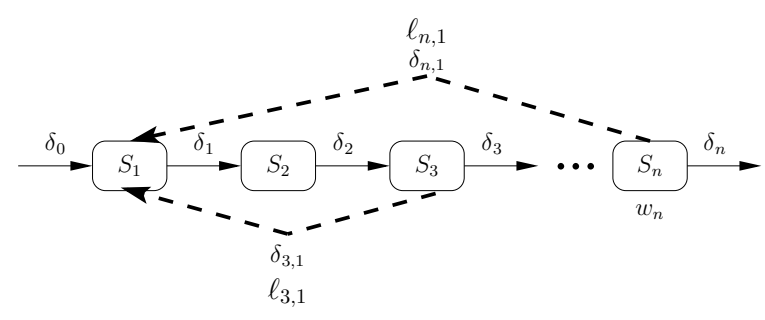

Fig. 5. Feedback loops.

There might be some dependencies between data sets for different stages, represented as feedback loops, see Figure 5 for an example with two feedback loops. An arrow going from stage $S_{k^{\prime}}$ to stage $S_{k}$, where $k^{\prime}>k$, and labeled with a positive integer $\ell_{k^{\prime}, k}$, means that $S_{k}$ needs the output from $S_{k^{\prime}}$ of data set $i-\ell_{k^{\prime}, k}$ to compute data set $i$. The size of data to be transferred along this feedback arrow is denoted as $\delta_{k^{\prime}, k}$. Such an arrow generates a loop in the application graph. For each feedback loop, the feedback data must arrive on time so that it is possible to perform the desired computation on the next data. A 
mapping will thus be valid only if there are not too many stage intervals (or processor changes) inside a loop.

Assume that there is a loop labeled with $\ell_{j, i}$, going from $S_{j}$ to $S_{i}$ (with $j>i$ ). As discussed in Section II-C, processor $a(i)$ is processing data set $\mathbf{d s}^{(k)}$ while sending data set $\mathbf{d s}{ }^{(k-1)}$ to the next processor in the line, which in the meantime processes data set $d \mathbf{s}^{(k-2)}$ and sends data set $\mathrm{ds} \mathbf{s}^{(k-3)}$, and so on. Thus, in order to get the data set on time, we need to ensure that $2 .\left(K(i, j)-1+\Delta_{a(i) \neq a(j)}\right) \leq \ell_{j, i}$, where $\Delta_{a(i) \neq a(j)}=1$ if $a(i) \neq a(j)$, and 0 otherwise.

For instance, consider the feedback loop from $S_{3}$ to $S_{1}$ in Figure 5. If stages $S_{1}$ and $S_{2}$ are mapped onto, say, processor $P_{1}$ while $S_{3}$ is mapped onto $P_{2}$, then $K=2, a(1)=1 \neq a(3)=2$, and the formula states that we must have $4 \leq \ell_{3,1}$. Indeed, when $P_{1}$ operates on data set ds ${ }^{(k)}, P_{2}$ operates on ds ${ }^{(k-2)}$ and sends data corresponding to $\mathrm{ds}^{(k-3)}$ back to $P_{1}$, just in time if $\ell_{3,1}=4$ for $P_{1}$ to compute ds ${ }^{(k+1)}$ during the next period. Note that if the whole interval from $S_{i}$ to $S_{j}$ is mapped onto the same processor, then $K=1$ and $\Delta_{a(i) \neq a(j)}=0$, hence we derive the constraint $0 \leq \ell_{j, i}$, which is fine because data is available on site for the next period.

Feedback loops not only impose constraints on the mapping. We also need to revisit the expression for the period that was given in Section II-C.2 to account for the additional communications induced by the feedback loops. Rather than going on formally, we just illustrate this with the previous example: with the same mapping, the feedback loop from $S_{3}$ to $S_{1}$ induces an additional input communication that must be added to the other incoming communications of $P_{1}$, and an additional output communication that must be added to the other outgoing communications of $P_{1}$.

The generalization of interval-based mappings when considering feedback loops are connected-subgraph mappings, in which each processor is assigned a connected subgraph instead of an interval. Thus, two stages linked with a feedback loop can be mapped on the same processor, so that the feedback communication is done locally.

\section{B. Infinite Number of Processors}

Theorem 7: On Fully Homogeneous platforms with an infinite number of processors, finding the general mapping which minimizes the period for a linear chain application graph with feedback loops is NP-complete. 
Proof: The reduction comes from 2-PARTITION [12]. Please refer to [4] for the proof.

Like period, interval mappings are not optimal for latency on infinite number of processors when the application has feedback loops. It seems unlikely that the mapping with minimum latency can be found in polynomial time, since this problem appears to be as difficult as the problem of minimizing latency while mapping on a finite number of processors. We have, however, not been able to prove that this problem is indeed NP-complete.

\section{Approximation Results}

Also, the approximation result for period on Fully Homogeneous platforms (Theorem 4) does not hold when adding feedback loops. We refer to [4] for a counterexample that shows that interval mapping cannot provide any constant-approximation for pipeline workflows with feedback loops.

However, the approximation result for latency on Communication Homogeneous platforms still holds for applications with feedback loops using the same proof given in Theorem 5 in Section V.

\section{CONCLUSION}

This work presents complexity results for mapping linear workflows with computation/communication overlap, under the bounded multiport model. We provide a formal definition of period and latency optimization problems for such linear workflows and prove several major results; in particular the NP-completeness of the period minimization problem even on Fully Homogeneous platforms. Latency becomes NP-complete as soon as platforms are Communication Homogeneous, and the complexity remains open for Fully Homogeneous platforms. We provide a 2-approximation algorithm for the period on Fully Homogeneous platforms and a 1.5-approximation algorithm for the latency on Communication Homogeneous platforms. For some special mapping rules (restricting to interval-based mappings) and special cases (infinite number of identical processors, regular applications), we succeed in deriving polynomial algorithms for Fully Homogeneous platforms. Also, we introduce the concept of feedback loops to provide control to linear workflows. Such feedbacks add a level of complexity to most previous problems, since some special cases become NP-complete, and approximation results for the period do not hold anymore.

We believe that this exhaustive study of complexity results provides a solid theoretical foundation for the study of linear workflow mappings, with or without feedback loops, under the bounded multiport 
model with overlap. As future work, we plan to design some efficient polynomial-time heuristics to solve the many combinatorial instances of the problem, and to assess their performance through extensive simulations. This can become challenging in the presence of feedback loops. Finally, it would be interesting to study workflows in a different context (like web-service applications [13]) where each stage $S_{i}$ has a selectivity $\sigma_{i}$ parameter which is the ratio between its input and output data $\delta_{i-1} / \delta_{i}$. In these problems, the application dag is not fixed, and the aim is to generate the dag and schedule it so as to decrease the period and/or latency. There may be some constraints on what types of dags are permissible. For instance, given two stages $S_{1}$ and $S_{2}$ with selectivities $\sigma_{1}<\sigma_{2}$, on a homogeneous platform, it is better to create a dag where $S_{1}$ precedes $S_{2}$ to minimize the period. We plan to generalize our results for these kinds of applications.

\section{REFERENCES}

[1] R. Stephens, "A survey of stream processing," Acta Informatica, vol. 34, no. 7, pp. 491-541, 1997.

[2] J. Subhlok and G. Vondran, "Optimal latency-throughput tradeoffs for data parallel pipelines," in ACM Symposium on Parallel Algorithms and Architectures SPAA'96. ACM Press, 1996, pp. 62-71.

[3] A. Benoit and Y. Robert, "Mapping pipeline skeletons onto heterogeneous platforms," J. Parallel Distributed Computing, vol. 68, no. 6, pp. 790-808, 2008.

[4] K. Agrawal, A. Benoit, and Y. Robert, "Mapping linear workflows with computation/communication overlap," LIP, ENS Lyon, France, Research Report 2008-21, June 2008, available at graal.ens-lyon.fr/ abenoit/.

[5] Y.-K. Kwok and I. Ahmad, "Static scheduling algorithms for allocating directed task graphs to multiprocessors," $A C M$ Computing Surveys, vol. 31, no. 4, pp. 406-471, 1999.

[6] P. Bhat, C. Raghavendra, and V. Prasanna, "Efficient collective communication in distributed heterogeneous systems," Journal of Parallel and Distributed Computing, vol. 63, pp. 251-263, 2003.

[7] T. Saif and M. Parashar, "Understanding the behavior and performance of non-blocking communications in MPI," in Proceedings of Euro-Par 2004: Parallel Processing, ser. LNCS 3149. Springer, 2004, pp. 173-182.

[8] S. Khuller and Y. Kim, "On broadcasting in heterogenous networks," in Proceedings of the fifteenth annual ACM-SIAM symposium on Discrete algorithms. Society for Industrial and Applied Mathematics, 2004, pp. 1011-1020.

[9] B. Hong and V. Prasanna, "Bandwidth-aware resource allocation for heterogeneous computing systems to maximize throughput," in Proceedings of the 32th International Conference on Parallel Processing (ICPP'2003). IEEE Computer Society Press, 2003.

[10] N. T. Karonis, B. Toonen, and I. Foster, "Mpich-g2: A grid-enabled implementation of the message passing interface," J.Parallel and Distributed Computing, vol. 63, no. 5, pp. 551-563, 2003.

[11] B. Kreaseck, L. Carter, H. Casanova, J. Ferrante, and S. Nandy, "Interference-aware scheduling," International Journal of High Performance Computing Applications, vol. 20, no. 1, pp. 45-59, 2006.

[12] M. R. Garey and D. S. Johnson, Computers and Intractability, a Guide to the Theory of NP-Completeness. W.H. Freeman and Company, 1979.

[13] U. Srivastava, K. Munagala, J. Widom, and R. Motwani, “Query optimization over web services," in VLDB '06: Proceedings of the 32nd Int. Conference on Very Large Data Bases. VLDB Endowment, 2006, pp. 355-366. 